\title{
Duodenal Ulcer, CTCAE
}

National Cancer Institute

\section{Source}

National Cancer Institute. Duodenal Ulcer, CTCAE. NCI Thesaurus. Code C143419.

A disorder characterized by a circumscribed, erosive lesion on the mucosal surface of the duodenal wall. 